\title{
Cancelamento de procedimentos cirúrgico ambulatoriais: Análise Descritiva em um Hospital de Referência entre 2016/2018
}

\author{
Cancellation of ambulatory surgical procedures: Descriptive Analysis in a Referral Hospital \\ between 2016/2018
}

\begin{abstract}
Cancelación de procedimientos quirúrgicos ambulatorios: Análisis descriptivo en un hospital de referencia entre 2016/2018
\end{abstract}

Francisco Alves Lima Júnior ${ }^{1,2,4,5 *}$, Edvan Ramos Vieira ${ }^{4}$, Karla Vanessa Morais Lima ${ }^{3,6}$, Raquel Machado Borges ${ }^{3}$, Denilson da Silva Lima ${ }^{3}$. Anne Caroline Almeida de Arruda Cruz ${ }^{5}$, Jeíse Pereira Rodrigues $^{2}$, Jannyelle Priscila Alves Brandão ${ }^{2}$, Janeide Pereira dos Santos de Gois ${ }^{6}$, Felype Hanns Alves de Medeiros ${ }^{2,5}$, Patrícia dos Santos Silva Quiroz ${ }^{4}$, Eduardo Morais da Silva ${ }^{4}$, Níbia Maria da Silva², Marcus Vinícius Henriques Brito².

\section{RESUMO}

Objetivo: Apresentar os principais motivos que levaram ao cancelamento cirúrgico em um hospital de referência em cirurgias ambulatoriais. Métodos: Trata-se de uma pesquisa do tipo descritiva, documental e retrospectiva, com abordagem quantitativa para o tratamento dos dados, onde se analisou 28 relatórios de produção do Centro Cirúrgico referente ao Período de outubro de 2016 a dezembro de 2018 de um hospital de referência em cirurgias de ambulatoriais. Resultados: Foram analisados 28 relatórios emitidos pela supervisão do Centro Cirúrgico, ao todo 8717 pacientes foram agendados para realização de cirurgias, destas apenas $8059(92,45 \%)$ foram realizadas, onde as $658(7,55 \%)$ foram canceladas devido aos fatores relacionados: $54,26 \%$ relacionados ao paciente, $15,2 \%$ a organização da unidade, $17,93 \%$ aos recursos humanos, 7,44\% recursos materiais e $5,17 \%$ a outros fatores. Conclusão: A investigação dos motivos de suspensões cirúrgicas traz benefícios à instituição, como na pesquisa em questão em que os fatores encontrados são mutáveis.

Palavras-chave: Procedimentos Cirúrgicos ambulatoriais, Centro Cirúrgico, Administração de Serviços de Saúde.

\begin{abstract}
Objective: to present the main reasons that led to the cancellation in a hospital ambulatory surgery reference. Methods: this is a descriptive research, documentary and retrospective, with quantitative approach for the treatment of the data, where he analyzed 28 production reports from the Surgical Center for the period of October to December 2016, 2018 of a reference hospital in outpatient surgeries. Results: we analyzed 28 reports issued by supervision of the Surgical Center, all patients were scheduled 8717 for performing these surgeries just 8059 (92.45\%) were held, where the 658 (7.55\%) were cancelled due to factors related: $54.26 \%$ related to patient, $15.2 \%$ the unit organization, $17.93 \%$ human resources, $7.44 \% 5.17 \%$ to material resources and other factors. Conclusion: the investigation of the reasons of the institution benefits surgical suspensions, as the research in question in which the factors found are changeable.
\end{abstract}

Keywords: Ambulatory Surgical, Surgicenters, Health Services.

1 Universidade Estadual do Tocantins - UNITINS, Augustinópolis - TO.

2Universidade do Estado do Pará, UEPA, Belém-PA.

${ }^{3}$ Faculdade de Imperatriz - FACIMPM WYDEN, Imperatriz - MA.

${ }^{4}$ Universidade Ceuma, campus Imperatriz-MA;

${ }^{5}$ Hospital Macrorregional Dra. Ruth Noleto, Imperatriz-MA.

6Universidade Federal do Maranhão. Imperatriz-MA.*E-mail: francisco.enfdotrabalho@gmail.com 


\section{RESUMEN}

Objetivo: presentar los principales motivos que llevaron a la cancelación en un hospital de referencia de cirugía ambulatoria. Métodos: se trata de una investigación descriptiva, documental y retrospectiva, con enfoque cuantitativo para el tratamiento de los datos, donde analizó 28 informes de producción del centro quirúrgico durante el período de octubre a diciembre 20162018 de un hospital de referencia en cirugías ambulatorias. Resultados: se analizaron 28 informes emitidos por la supervisión del centro quirúrgico, todos los pacientes fueron programados 8717 para realizar estas cirugías sólo 8059 (92.45\%) se llevaron a cabo, donde la $658(7,55 \%)$ fueron cancelados debido a factores relacionados con: $54.26 \%$ relacionados con paciente, $15.2 \%$ la organización de la unidad, recursos humanos de $17.93 \%, 7,44 \% 5.17 \%$ a recursos materiales y otros factores. Conclusión: la investigación de las razones de la institución beneficia a suspensiones quirúrgicas, como la investigación en cuestión en la que los factores encontraron son cambiables.

Palabras clave: Procedimientos Quirúrgicos, Centros Quirúrgicos, Administración de los Servicios de Salud.

\section{INTRODUÇÃO}

Sabe-se que o processo de adoecimento envolve múltiplos fatores para sua determinação, sendo assim, faz-se necessárias a tomada de intervenções que atuem como refratores ou controladores de certos eventos relacionados a saúde da população, para tanto, necessita-se de intervenções eficazes nos mais diferentes níveis de atenção à saúde, contudo, diante da concentração existente dos serviços de saúde, uma medida tem sido adotada para garantir a continuidade do processo de intervenção em saúde que é o processo de referência e contra referência (NETO JF, et al., 2018).

Devido ao esgotamento de recursos assistenciais encontrado na atenção primaria algumas ações devem ser tomadas para garantia da continuidade do atendimento ao paciente, em que, ao encontra-se mediante a um processo patológico que necessite de uma intervenção cirúrgica o mesmo deverá ser encaminhado a um serviço assistencial de nível superior como a atenção hospitalar, neste caso, encontra-se as cirurgias ambulatoriais, no qual, estas constituem-se de importantes arranjos de acompanhamento de qualidade aos pacientes (SANTOS MC, 2015; RAMOS PLC, 2018).

Para tanto, conceitua-se as cirurgias ambulatoriais como procedimentos cirúrgicos para fins curativos com dispensa da internação do paciente, sendo estes realizados em um ambiente propicio, neste caso ocorrera no Bloco Cirúrgico ou Centro Cirúrgico (CC), no qual, seu período de permanência na instituição não ultrapassa usualmente às 12 horas, período este que após seu termino já poderia ser considerado como uma internação (SAMPAIO CEP e RIBEIRO DA, 2012).

O Centro Cirúrgico é o local onde deve ser provido de recursos tecnológicos preparados para suprir as adversidades que surgem na unidade, esses equipamentos devem fornecer suporte amplo na sala cirúrgica, para a qual o cliente é submetido a procedimentos invasivos, e que, essa intervenção se faz necessários, para manter a vida desse indivíduo (FIGUEIREDO NMA, et al., 2006).

O CC é um setor complexo de entrada restrita, pertencente a uma instituição hospitalar e, é considerado um ambiente de maior complexidade dentro da magnitude de suas funcionalidades. Por possuir uma estrutura que deve seguir à legislação sanitária vigente e seguir diretrizes de normas técnicas criadas pela vigilância sanitária local. A cirurgia sempre foi realizada dentro das salas cirúrgicas independentemente de sua complexibilidade, mesmo as ambulatoriais consideradas de baixo risco operatório (MARTIN ARG, 2012).

Atualmente no CC atuam profissionais de diversas especialidades, com formação, experiência e objetivos diferentes, mas que mesmo assim devem trabalhar em harmonia. Todavia, é neste ambiente complexo e multifuncional que ocorrem adversidades que levam a comunicações errôneas, causas conflitantes, e erros impactantes, não de inabilidade técnica, mas de péssimas habilidades interpessoais entre a equipe (LOT JGM, 2015).

Os profissionais pioneiros na cirurgia detinham pouco conhecimento dos processos de assepsia, e os métodos de anestesia eram ultrapassados e não mostravam confiabilidade. Em meados de 1840, foi 
descoberta a anestesia, favorecendo o início de avanços nas práticas operatórias. O processo anestésico ampliou a fusão entre analgesia, relaxamento musculoesquelético e amnesia dos sentidos, possibilitando 0 aumento do tempo para realização da cirurgia (MOREIRA LR, et al., 2016).

Embora se reconheça a necessidade da intervenção cirúrgica em alguns casos ainda assim ocorrem-se alguns fatores que levam ao cancelamento do procedimento, para tanto este projeto tem por finalidade apresentar os principais motivos que levaram ao cancelamento cirúrgico em um hospital de referência em cirurgias ambulatoriais.

\section{MÉTODOS}

\section{Descrever a metodologia de forma mais detalhada, critérios de inclusão, exclusão}

Trata-se de uma pesquisa do tipo descritiva, documental e retrospectiva, na qual, empregou-se uma abordagem quantitativa para o tratamento dos dados obtidos, sendo estes provenientes da pesquisa de campo, da qual se analisou 28 relatórios de produção do setor de Centro Cirúrgico referente ao Período de outubro de 2016 a dezembro de 2018 de um Hospital de Referência Cirúrgica no Sul do Maranhão, sendo tal instituição caracterizada como um hospital de referência em cirurgias de ambulatoriais.

A fim de se obter êxito na realização do objetivo proposto à pesquisa os dados colhidos na foram catalogados e trabalhos estatisticamente sobre o programa Microsoft Exce/® 2010, bem como, representados graficamente segundo tal programa, com posterior discussão das evidencias encontradas na pesquisa segundo a literatura vigente.

\section{RESULTADOS}

Foram analisados 28 relatórios emitidos pela supervisão do Centro Cirúrgico, verificando ao todo 8717 pacientes, que foram agendados para realização de procedimentos cirúrgicos, destas apenas 8059 (92,45\%) cirurgias foram realizadas de todas as especialidades médicas, as $658(7,55 \%)$ foram canceladas devido aos fatores relacionados ao paciente, a organização da unidade, recursos humanos, recursos materiais, dentre outros. O Gráfico 1 mostra que a maioria dos cancelamentos ou suspensão de procedimentos se devem a motivos relacionados ao paciente, com $357(54,26 \%)$ suspensões, seguido de recursos humanos com 117 $(17,93 \%)$, organização da unidade com 100 (15,2\%), materiais e equipamentos com $49(7,44 \%)$ e outros motivos não explicitados com 35 (5,17\%) cancelamentos / suspensões.

Gráfico 1 - Distribuição dos principais motivos de suspensões cirúrgicas do Hospital Macrorregional de Imperatriz - MA entre outubro de 2016 a dezembro de 2018.

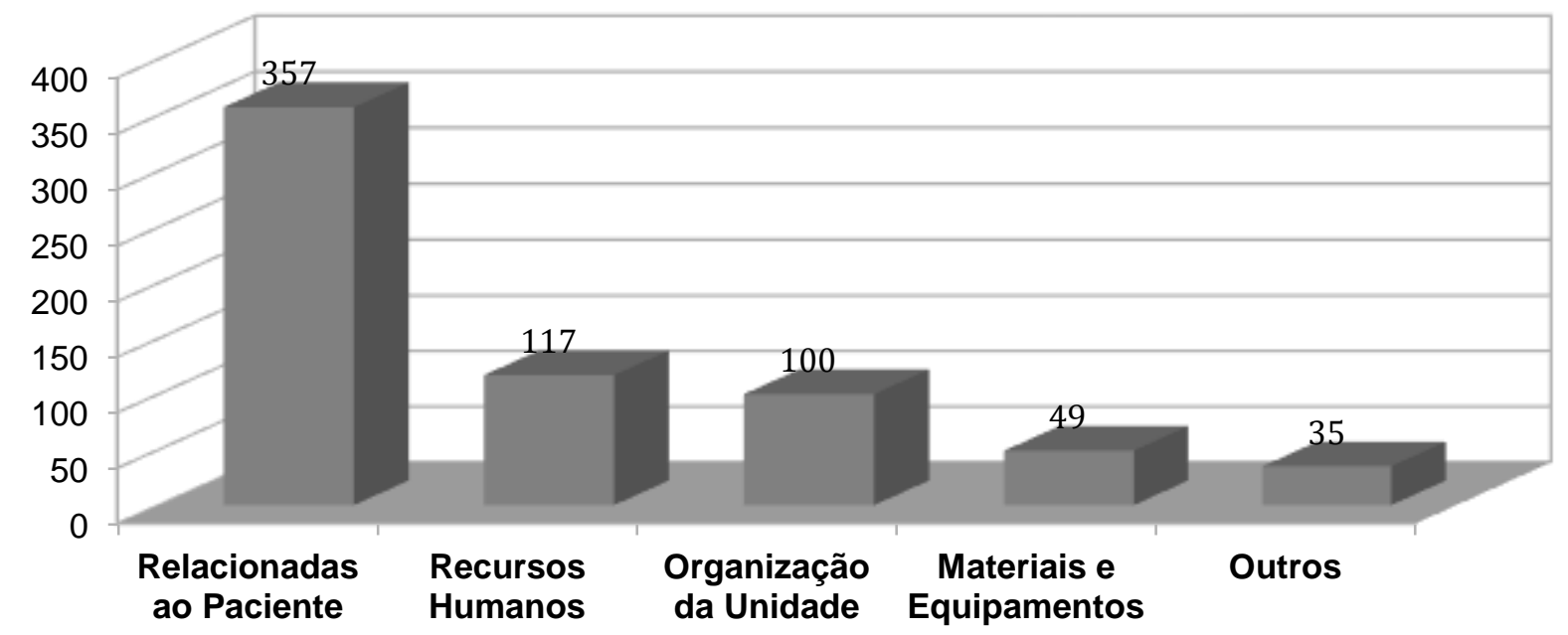

Fonte: Lima Júnior FA, et al., 2019. 
Dentre os principais motivos que levaram a suspensão das cirurgias ambulatoriais relacionadas ao paciente evidenciou-se: $150(22,8 \%)$ devido ao não comparecimento do paciente, $193(29,33 \%)$ a condição clinica dos mesmos, $2(0,3 \%)$ a não realização do jejum, $9(1,37 \%)$ a recusa quanto a realização do procedimento, e por fim $3(0,46 \%)$ devido ao óbito do paciente antes que o procedimento tenha sido realizado, conforme apresentado na Tabela 1.

Tabela 1 - Subdivisão das suspensões cirúrgicas ambulatoriais Relacionadas ao Paciente do Hospital Macrorregional de Imperatriz - MA entre outubro de 2016 a dezembro de 2018.

\begin{tabular}{cccccccccc}
\hline PERÍODO & \multicolumn{2}{c}{$\mathbf{2 0 1 6}$} & \multicolumn{2}{c}{$\mathbf{2 0 1 7}$} & \multicolumn{2}{c}{$\mathbf{2 0 1 8}$} & \multicolumn{2}{c}{ TOTAL } \\
QUANTIDADE / \% & $\mathbf{N}^{\circ}$ & $\%$ & $\mathbf{N}^{\circ}$ & $\%$ & $\mathbf{N}^{\circ}$ & $\%$ & $\mathbf{N}^{\circ}$ & $\%$ \\
\hline Não compareceu & 14 & 2,13 & 65 & 9,88 & 71 & 10,79 & 150 & 22,8 \\
Cond. Clínica & 31 & 4,71 & 110 & 16,72 & 52 & 7,9 & 193 & 29, \\
Falta Jejum & 0 & 0 & 0 & 0 & 2 & 0,3 & 2 & 0,3 \\
Recusa na realização & 0 & 0 & 5 & 0,76 & 4 & 0,61 & 9 & 1,37 \\
Óbito do paciente & 0 & 0 & 0 & 0 & 3 & 0,46 & 3 & 0,46 \\
\hline TOTAL & $\mathbf{4 5}$ & $\mathbf{6 , 8 4}$ & $\mathbf{1 8 0}$ & $\mathbf{2 7 , 3 6}$ & $\mathbf{1 3 2}$ & $\mathbf{2 0 , 0 6}$ & $\mathbf{3 5 7}$ & $\mathbf{5 4}$ \\
\hline
\end{tabular}

Fonte: Lima Júnior FA, et al., 2019.

Na Tabela 2 podemos acompanhar os principais motivos de cancelamento cirúrgico relacionado aos recursos humanos, evidenciados na pesquisa.

Tabela 2 - Subdivisão das suspensões cirúrgicas ambulatoriais relacionadas aos Recursos Humanos do Hospital Macrorregional de Imperatriz - MA entre outubro de 2016 a dezembro de 2018.

\begin{tabular}{lllllllll}
\hline PERÍODO & $\mathbf{2 0 1 6}$ & \multicolumn{3}{c}{$\mathbf{2 0 1 7}^{\circ}$} & \multicolumn{2}{c}{$\mathbf{2 0 1 8}$} & \multicolumn{3}{c}{ TOTAL } \\
QUANTIDADE/\% & $\mathbf{N}^{\circ}$ & $\%$ & $\mathbf{N}^{\circ}$ & $\%$ & $\mathbf{N}^{\circ}$ & $\%$ & $\mathbf{N}^{\circ}$ & $\%$ \\
\hline Falta de Leito de UTI & 0 & 0 & 6 & 0,91 & 4 & 0,61 & 10 & 1,52 \\
Cancelamento do Cirurgião & 9 & 1,37 & 63 & 9,57 & 21 & 3,19 & 93 & 14,13 \\
Cirurgião não compareceu & 0 & 0 & 0 & 0 & 5 & 0,76 & 5 & 0,76 \\
Mudança de Conduta Médica & 0 & 0 & 0 & 0 & 4 & 0,61 & 4 & 0,61 \\
Falta de Preparo & 0 & 0 & 3 & 0,46 & 3 & 0,46 & 6 & 0,92 \\
\hline TOTAL & $\mathbf{9}$ & $\mathbf{1 , 3 7}$ & $\mathbf{7 2}$ & $\mathbf{1 0 , 9 4}$ & $\mathbf{3 7}$ & $\mathbf{5 , 6 3}$ & $\mathbf{1 1 8}$ & $\mathbf{1 7 , 9 4}$ \\
\hline
\end{tabular}

Fonte: Lima Júnior FA, et al., 2019.

Com base neste levantamento acerca do assunto a Tabela 3 traz consigo as informações referentes aos fatores relacionados ao cancelamento cirúrgico devido a causas de caráter gerencial da instituição.

Tabela 3 - Subdivisão das suspensões cirúrgicas ambulatoriais relacionadas ao sistema gerencial do Hospital Macrorregional de Imperatriz - MA entre outubro de 2016 a dezembro de 2018.

\begin{tabular}{lllllllll}
\hline PERÍODO & \multicolumn{2}{l}{2016} & \multicolumn{2}{c}{2017} & \multicolumn{2}{c}{$\mathbf{2 0 1 8}$} & \multicolumn{3}{c}{ TOTAL } \\
QUANTIDADE/\% & $\mathbf{N}^{\circ}$ & $\%$ & $\mathbf{N}^{\circ}$ & $\%$ & $\mathbf{N}^{\circ}$ & $\%$ & $\mathbf{N}^{\circ}$ & $\%$ \\
\hline Erro de agendamento & 7 & 1,07 & 27 & 4,1 & 31 & 4,71 & 65 & 9,88 \\
Tempo cirurgia excedida & 0 & 0 & 0 & 0 & 1 & 0,15 & 1 & 0,15 \\
Falta de exame & 1 & 0,15 & 24 & 3,65 & 9 & 1,37 & 34 & 5,17 \\
\hline TOTAL & $\mathbf{8}$ & $\mathbf{1 , 2 2}$ & $\mathbf{5 1}$ & $\mathbf{7 , 7 5}$ & $\mathbf{4 1}$ & $\mathbf{6 , 2 3}$ & $\mathbf{1 0 0}$ & $\mathbf{1 5 , 2}$ \\
\hline
\end{tabular}

Fonte: Lima Júnior FA, et al., 2019. 
Além dos fatores supracitados encontram-se como possíveis causadores de suspensão da cirurgia os fatores relacionados aos recursos materiais, neste caso a (Tabela 4) evidencia os fatores relacionados aos recursos materiais, bem como os fatores não identificados que levaram a suspensão dos procedimentos cirúrgicos no local de pesquisa.

Tabela 4 - Subdivisão das suspensões cirúrgicas ambulatoriais relacionadas aos recursos materiais e fatores não identificados do Hospital Macrorregional de Imperatriz - MA entre outubro de 2016 a dezembro de 2018.

\begin{tabular}{lllllllll}
\hline PERÍODO & 2016 & \multicolumn{2}{c}{2017} & \multicolumn{2}{c}{$\mathbf{2 0 1 8}$} & \multicolumn{3}{c}{ TOTAL } \\
QUANTIDADE/\% & $\mathbf{N}^{\circ}$ & $\%$ & $\mathbf{N}^{\circ}$ & $\%$ & $\mathbf{N}^{\circ}$ & $\%$ & $\mathbf{N}^{\circ}$ & $\%$ \\
\hline Falta de & 2 & 0,3 & 35 & 5,32 & 12 & 1,82 & 49 & 7,44 \\
Equipamentos & 0 & 0 & 17 & 2,58 & 17 & 2,58 & 34 & 5,16 \\
Motivos Não Informados & $\mathbf{2}$ & $\mathbf{0 , 3}$ & $\mathbf{5 2}$ & $\mathbf{7 , 9}$ & $\mathbf{2 9}$ & $\mathbf{4 , 4}$ & $\mathbf{8 3}$ & $\mathbf{1 2 , 6}$ \\
\hline TOTAL & & & & & & &
\end{tabular}

Fonte: Lima Júnior FA, et al., 2019.

No ano de 2017, houve um grande aumento no cancelamento de cirurgias ambulatoriais relacionadas principalmente por: condições clínicas do paciente 110 (16,72), cancelamento do cirurgião 63 (9,57\%), falta de exames laboratoriais 24 (3,65\%) e também de equipamentos ou materiais 35 (5,32\%), mas em 2018 esses valores regrediram drasticamente totalizando o ano com apenas $94(14,28 \%)$ desses tipos de cancelamentos.

\section{DISCUSSÃO}

Em um estudo realizado pela Universidade de Brasília que observou o processo cirúrgico eletivo em um Centro Cirúrgico de Referência, mostrou que das 171 (18\%) das cirurgias ambulatoriais canceladas estavam relacionadas com o paciente, já $37 \%$ destas a organização da unidade, $29 \%$ por outras causas, relacionadas aos recursos humano $29 \%$, aos equipamentos e materiais $2 \%$, e $2 \%$ canceladas por causas não especificadas (BITENCOURT JA, 2018).

Em outro estudo, realizado em um Hospital Universitário da cidade de são Paulo, mostrou que das 934 cirurgias programadas a nível ambulatorial, apresentaram no geral um quantitativo de 186 suspensões cirúrgicas, representando desse modo $19,91 \%$ dos resultados consolidados de procedimentos da instituição (SOUZA NVDO, et al., 2010).

Estudos sobre cancelamento de cirurgias eletivas apontam elevadas taxas que variam de $17,6 \%$ a $33 \%$, sendo que, a ocorrência de suspensão cirúrgica deve ser um aspecto investigado, buscando minimizá-las, pois a não realização operatória pode trazer dificuldades psicossociais para lidar com os sentimentos do paciente, e que pode ser agravada devido às peculiaridades de cada indivíduo (SAMPAIO CEP e RIBEIRO DA, 2012).

Em uma pesquisa realizada no Hospital de Grande Porte em belo Horizonte, Minas Gerais, mostrou que dos 5746 procedimentos cirúrgicos agendados, 298 foram cancelados, evidenciando assim uma taxa de suspensão de $5,2 \%$, no qual, os principais motivos das suspensões encontrados na pesquisa foram: falta de autorização $51,5 \%$ e o paciente sem condições clínicas $19,5 \%$, onde se percebe que os motivos de cancelamentos das cirurgias estão relacionados a problemas administrativos e a existência de lacunas nas ações direcionadas aos clientes (MOREIRA LR, et al., 2016).

Apesar de existir vários cancelamentos de cirurgias no hospital pesquisado, estudos mostram que o índice de suspensão cirúrgico ambulatorial no quantitativo geral encontra-se dentro da média quando comparado ás pesquisas realizadas e publicadas em outras instituições, além disto, artigos de revistas e jornais publicados na Espanha, Austrália, Inglaterra, Nigéria, Jordânia e aqui no Brasil, mostraram em seus estudos diversas taxas de cancelamento de procedimento cirúrgico a nível ambulatorial que variam entre $3,6 \%$ a $28 \%$ (SAMPAIO CEP e RIBEIRO DA, 2012). 
Na verdade, não deveria existir cancelamento de cirurgia, todavia, sabem-se que diversas situações são pertinentes as medidas tomadas pelo o hospital, como é o caso de clientes que apresentaram condições clínicas desfavoráveis após a avaliação e preparo pré-operatório, neste caso, torna-se preciso reduzir as situações imprevistas ou que divergem do planejamento preliminar, corroborando assim, para redução desses eventos tão prejudiciais aos pacientes/família, equipe e instituição (CHANG JH, et al., 2014).

$\mathrm{Na}$ pesquisa realizada na China Medical University Hospital entre 2006 a 2011, constatou que os cancelamentos pelo profissional cirurgião se mostraram em $277(67,1 \%)$ das operações canceladas, e que, foram realizadas em um momento posterior (acabaram realizando a cirurgia, mas após algum tratamento complementar). Esses dados sugerem que alguns dos motivos de suspensões devem ser modificados alterados ou evitados, mas que a cirurgia suspensa foi necessária (PERROCA MG, et al., 2007).

Em um estudo realizado em 2007, evidenciou-se que 53\% dos cancelamentos que ocorreram após a sala de operação ter sido preparada para a cirurgia estavam relacionados ao paciente, sendo que, a maioria dos cancelamentos $(29 \%)$ foi devido à condição médica do paciente e $24 \%$ foram devidos a problemas organizacionais, como por exemplo, à falta de leitos de UTI (MIRANDA AB, et al., 2016).

Observa-se uma ineficiência de leitos em Salas de recuperação Pós-Anestésicas (SRPA) institucionais com o aumento da demanda de pacientes, assim, ocasionando uma retenção do paciente por período de tempo superior ao necessário, aumentando riscos de incidentes, tais como quedas, lesão de pele, fraturas, ocasionado devido a posição de vulnerabilidade, justificando assim o cancelamento de outros procedimentos.(EZIKE HA, et al., 2011).

Em um relatório feito na Nigéria, Índia, e Arábia Saudita, indisponibilidade de leitos de cirurgias na Unidade de Terapia Intensiva foram os principais motivos para o cancelamento de cirurgias, e a subestimação do tempo cirúrgico de longa duração foi uma das razões que ocasionaram a falta de salas cirúrgicas (GARG R, et al., 2009; EZIKE HA, et al., 2011; KUMAR R e GANDHI R, 2012; SULTAN N. et al., 2012).

Muitos dos cancelamentos cirúrgicos são devido à má alocação de instalações hospitalares ou ainda a falta de avaliação prévia na comunicação com o paciente. Em um estudo sobre cancelamentos de cirurgias em um hospital que aumentou sua capacidade de sala de operação, mas não fez quaisquer outras alterações, a suspensão devida à falta de leitos pós-operatórios aumentaram (PERROCA MG, et al., 2007; YOON SZ, et al., 2009).

\section{CONCLUSÃO}

A investigação dos motivos de suspensões cirúrgicas podem trazer benefícios no que diz respeito ao controle dos cancelamentos de procedimentos, podendo oferecer redução dessa taxa, além dos avanços na assistência e na eficiência da assistência cirúrgica prestada, uma vez que, a descrição, distribuição e avaliação dos dados possibilitam a percepção acerca dos motivos de cancelamento das cirurgias na instituição estudada, que estão, em sua maioria, relacionados aos problemas administrativos e de gerenciamento e da existência de lacunas nas ações direcionadas ao paciente.

\section{REFERÊNCIAS}

1. BITENCOURT JA. Análise do Processo de Cirurgias Eletivas em um Centro Cirúrgico de Referência: Potencializando as Atividades que Agregam Valor ao Paciente com Base em Princípios Enxutos. Trabalho de conclusão de curso (graduação em engenharia de produção) Universidade de Brasília, 2018.

2. CHANG JH, et al. Case review analysis of operating room decisions to cancel surgery. BMC Surgery, [s.I.], v. 14, n. 1, p.2-6, 23 jul. 2014. Springer Nature. http://dx.doi.org/10.1186/1471-2482-14-47.

3. EZIKE HA, et al. Reasons for Elective Surgery Cancellation in a Referral Hospital. Annals of Medical and Health Sciences Research, Nigéria, v. 01, n. 02 p.197-202, 2011.

4. FIGUEIREDO NMA, et al. Centro Cirúrgico: Atuação, Intervenção e Cuidados de Enfermagem. Yendis, São Caetado do Sul, 2006.

5. GARG, R. et al. Reasons for Cancellation of Cases on the Day of Surgery-a Prospective Study. Indian Journal of Anaesthesia, Indian, v. 53, n. 01, p. 35-39, 2009. 
6. KUMAR R, GANDHI R. Reasons for cancellation of operation on the day of intended surgery in a multidisciplinary 500 bedded hospital. Journal of Anaesthesiology Clinical Pharmacology, [s.I.], v. 28, n. 1, p.1-66, nov. 2012.

7. LOT JGM. Estudo dos Processos de Handoffs: um Caso do Centro Cirúrgico Ambulatorial do Hospital Universitário $\begin{array}{lllll}\text { de Brasília. } & \text { p. } & 1-78, & \text { Disponível }\end{array}$ <http://bdm.unb.br/bitstream/10483/11733/1/2015_JoaoGabrielMilhomemLot.pdf>.

8. MARTIN ARG. Critérios para a Implantação de uma Unidade Cirúrgica para Procedimentos de Alta Complexidade : Uma Visão Mutidisciplinar. Trabalho de conclusão de curso (Mestrado em Ciências da Saúde). Faculdade de Ciencias Médicas da Santa Casa De São Paulo, 2012.

9. MIRANDA AB, et al. Posicionamento Cirúrgico: Cuidados de Enfermagem no Transoperatório. Revista SOBECC, v. 21, n. 1, p.52-58, 8 jun. 2016.

10. MOREIRA LR, et al. Avaliação dos Motivos de Cancelamento de Cirurgias Eletivas. Revista de Enfermagem, p. 212225, 2016. Disponível em: <http://periodicos.pucminas.br/index.php/enfermagemrevista/article/view/13156>.

11. NETO JF, et al. Comunicação entre médicos a partir da referência e contra referência: potencialidades e fragilidades. CIAIQ2018, v. 2, 2018.

12. PERROCA MG, et al. Surgery cancelling at a teaching hospital: implications for cost management. Revista Latinoamericana de Enfermagem, v. 15, n. 5, p.1018-1024, 2007.

13. RAMOS PLC. Referência e contra referência no SUS: revisão integrativa da literatura. 27 f. Trabalho de conclusão de curso (Bacharelado em saúde coletiva). Universidade de Brasília, Brasília, 2018.

14. SAMPAIO CEP. RIBEIRO DA. Perfil Cirúrgico e Fatores Determinantes das Suspensões de Cirurgias Gerais Ambulatoriais: Contribuições para Assistência de Enfermagem. Rev Pesq Cuidado Fund On Line, v. 4, n. 2, p. 29382947, 2012.

15. SANTOS MC. Sistema de Referência e Contra referência em saúde em São Sebastião da Vitória, distrito de São João Del Rei-MG: O papel da rede na atenção básica. 32 f. Trabalho de conclusão de curso (especialização em Estratégia Saúde da Família) Universidade Federal de Minas Gerais. Minas Gerais, 2015.

16. SOUZA NVDO, et al. Determinantes para Suspensões Cirúrgicas em um Hospital Universitário. Revista Mineira de Enfermagem, p. 82-87, 2010. Disponível em: <http://reme.org.br/artigo/detalhes/91>.

17. SULTAN N, et al. Reasons for cancellation of elective cardiac surgery at Prince Sultan Cardiac Centre, Saudi Arabia. Journal of The Saudi Heart Association, [s.I.], v. 24, n. 1, p.29-34, jan. 2012.

18. YOON SZ, et al. The Effect of Increasing Operating Room Capacity on Day-of-surgery Cancellation. Anaesthesia And Intensive Care, [s.I.], v. 37, n. 2, p.261-266, mar. 2009. SAGE Publications. 